\title{
A MICROSTRUCTURE INVESTIGATION OF THE DEFORMATION AND RECRYSTALLIZATION OF PARTICLE-CONTAINING ALUMINIUM-SILICON ALLOYS
}

\author{
F. HABIBY $\dagger$ and F. J. HUMPHREYS $\ddagger$ \\ † Department of Materials, Imperial College, London SW72BP, England (now at \\ A. Q. Khan Research Laboratory, PO box 502, Rawalpindi, Pakistan) \\ $\ddagger$ Manchester Materials Science Centre, Grosvenor Street, Manchester M1 7HS, \\ England
}

\begin{abstract}
Single crystals and polycrystals of aluminium containing non-deformable second-phase particles of silicon, have been deformed, and the resultant structures investigated by microscopy and by X-ray and microtexture techniques. The particle size is found to influence the scale of the deformation bands formed, and there is evidence that particles may affect the nucleation of these bands. The deformed materials were recrystallized, and the effect of particle stimulated nucleation on the weakening of the rolling texture is discussed with reference to a computer simulation. In contrast, the recrystallization texture of particle-containing single crystals deformed on only two slip systems is sharp, and it is shown that the texture components are consistent with plasticity theory.
\end{abstract}

KEY WORDS Al-Si alloys, microtexture, deformation bands, particle stimulated nucleation.

\section{INTRODUCTION}

Despite extensive research, there are many aspects of the effects of second-phase particles on deformation and annealing that remain unclear. These include the effect of particles on large scale microstructural heterogeneities such as deformation bands and shear bands, and the effect of particle stimulated recrystallization on texture. The present work has been carried out on model alloys in order to investigate these effects.

\section{MATERIALS AND METHODS}

\section{Materials}

An alloy of aluminium containing $0.8 \mathrm{wt} \% \mathrm{Si}$ was chill cast as rods of $15 \mathrm{~mm}$ diameter. Rectangular specimens for cold rolling, were machined from the rods and heat treated to produce predominantly equiaxed precipitates of $\mathrm{Si}$ of various sizes. The heat treatments used were based on those of Humphreys (1977) and consisted of a solution treatment at $580^{\circ} \mathrm{C}$ followed by a cold water quench. Fine, uniform precipitation was induced by an anneal at $250^{\circ} \mathrm{C}$. The precipitates were 
Table 1 The materials used in the investigation

\begin{tabular}{lllll}
\hline $\begin{array}{l}\text { Specimen } \\
\text { type }\end{array}$ & Wt\% Si & $\begin{array}{l}\text { Particle diameter } \\
(\mu \mathrm{m})\end{array}$ & $\begin{array}{l}\text { Volume fraction } \\
\left(\times 10^{-3}\right)\end{array}$ & Code \\
\hline Polycrystal & 0.8 & 1.6 & 6.9 & PCS \\
Polycrystal & 0.8 & 6.6 & 6.9 & PCL \\
Single crystal & 0.8 & 0.7 & 6.9 & SCSx \\
Single crystal & 0.8 & 5 & 6.9 & SCLx $^{\mathrm{a}}$ \\
\hline a $\mathrm{x}$ denotes the crystal orientation. & & &
\end{tabular}

then grown by a further anneal at high temperature $\left(400^{\circ}\right.$ or $\left.480^{\circ} \mathrm{C}\right)$, and the specimens were slowly cooled to avoid any further nucleation of precipitates. Details of the particle parameters are given in Table 1. The grain size of the polycrystals was $500 \mu \mathrm{m}$.

Single crystals of various orientations were grown, and compression specimens of diameter $6 \mathrm{~mm}$ and length $12 \mathrm{~mm}$ were cut from these. The single crystals were heat treated to produce particles in the manner described above. The orientation of the single crystals is shown in Figure 2.

Previous work (Humphreys, 1977 and Humphreys, 1979) has shown that the particles do not deform or fracture during the subsequent deformation of the samples. Therefore these particles, which are contained in a low alloy matrix $(\mathrm{Al}-0.1 \% \mathrm{Si})$ provide a reasonably ideal model system.

\section{Experimental Methods}

Deformation was carried out in compression in a screw driven Instron testing machine at room temperature at a strain rate of $10^{-4} \mathrm{~s}^{-1}$. Specimens were also cold rolled to various reductions between $150 \mathrm{~mm}$ rolls, operating at $100 \mathrm{rpm}$.

Specimens were subsequently sectioned parallel to the compression axis, or perpendicular to the rolling plane by spark erosion, and were examined optically in plane polarised light after anodising, by SEM, using backscattered electron imaging, and by transmission electron microscopy. Bulk texture measurements were made by X-ray diffraction using a Philips Schulz-type diffractometer. Microtexture measurements were made by using the electron backscatter (EBSP) technique (Dingley 1987).

\section{DEFORMATION}

\section{Texture}

The 111 polefigures of specimens containing particles of different sizes, cold rolled by $85 \%$ are shown in Figure 1 . The textures are typical of cold rolled aluminium, and are not much weaker than those of single-phase aluminium (e.g. AA 1050) rolled to similar reductions.

Non-deformable second-phase particles generally weaken the deformation texture without adding any specific new components (Jensen et al., 1987). This is thought to be due to the deformation zones close to the particles within which the 


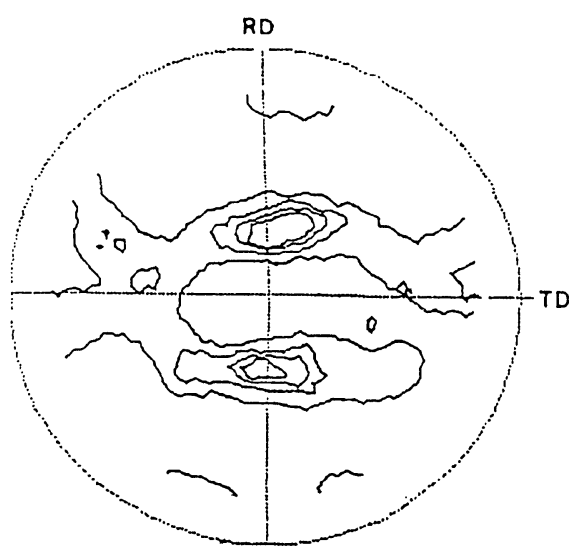

(a)

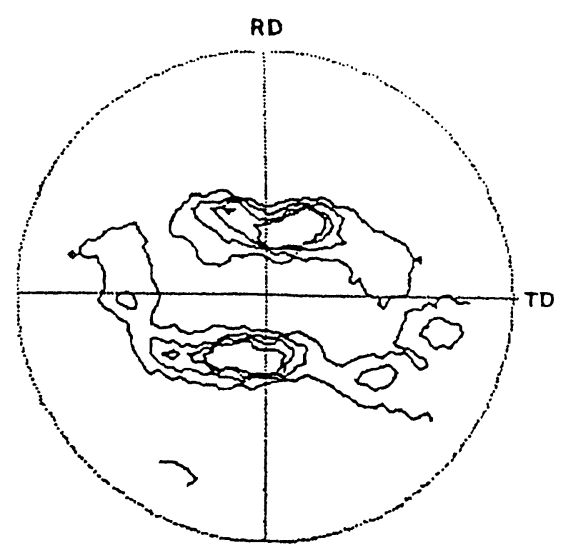

(b)

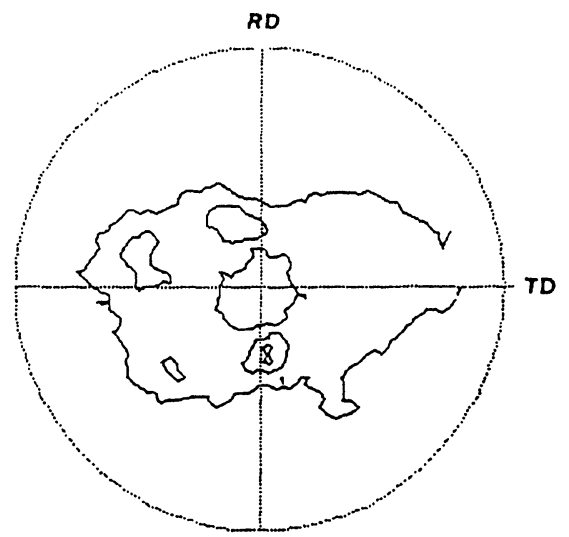

(c)

Figure 1111 pole figures from mid-section of polycrystals cold rolled $85 \%$. Contours are plotted of intervals of $1 \times$ random. a) Alloy PCL containing $6.6 \mu \mathrm{m}$ particles. b) Alloy PCS containing $1.6 \mu \mathrm{m}$ particles. c) PCL after recrystallization.

material is rotated (Humphreys and Kalu, 1990). As the volume fraction of particles is small in these specimens, the volume of the deformation zones is small and therefore the effect on the deformation texture is very limited.

\section{Microstructure}

Transmission electron microscopy of the microstructures developed close to the particles in similar materials has been reported previously (Humphreys, 1977, 1979). In this paper, we concern ourselves with the effect of the particles on the larger scale heterogeneities in the microstructure. It is well known that grains within a polycrystal do not deform homogeneously, but that they "break up" into regions of different orientation, known as deformation bands. This is thought to 
be a consequence of the different rotation paths followed by regions of the grain within which different combinations of slip systems operate (Dillamore and Katoh, 1974). In a similar manner, single crystals of unstable orientations will, on deformation, tend to split into regions of different orientation (Becker et al., 1991).

Although the general principles of the formation of deformation bands follow from crystal plasticity theory, the factors governing the scale of this fragmentation are not so clear. As shown schematically in Figure 3, a crystal or grain of a certain orientation (a) may split into two orientation components during deformation, but whether these bands will be coarse (b) or fine (c) is not obvious.

We have examined the effect of particles on the scale of deformation band formation by examining the behaviour of single crystals of identical orientation, but containing particles of different sizes. The initial orientation of the compression axes of the crystals is shown in Figure 2.

The microstructures of Figure 4 show the profound effect of particle size on the scale of the deformation bands. In the crystal with small particles $(d=0.7 \mu \mathrm{m})$ the deformation bands are coarse, as shown in Figure $4 \mathrm{~b}$, with a typical spacing of $100 \mu \mathrm{m}$, and the spacing is very similar to that in the single-phase alloy. However, in the crystal containing larger particles $(d=5 \mu \mathrm{m})$, the bands are an order of magnitude finer as shown in Figure 4a.

The orientations in the region of the deformation bands in the crystals was studied by means of electron backscatter patterns. Figure $5 \mathrm{a}$ is an electron micrograph of deformation bands in a crystal of orientation 2 , containing small particles (SCS2). The orientation of the matrix measured during a traverse along the marked line is shown in Figure 5b. The orientation angles presented in Figure $5 \mathrm{~b}$ are given relative to a reference orientation, and were obtained from the measurement of angle/axis pairs. The orientation results clearly identify the structures as being deformation bands with alternate misorientations of around $30^{\circ}$. Figure $6 \mathrm{a}$ shows a micrograph and the corresponding orientation measure-

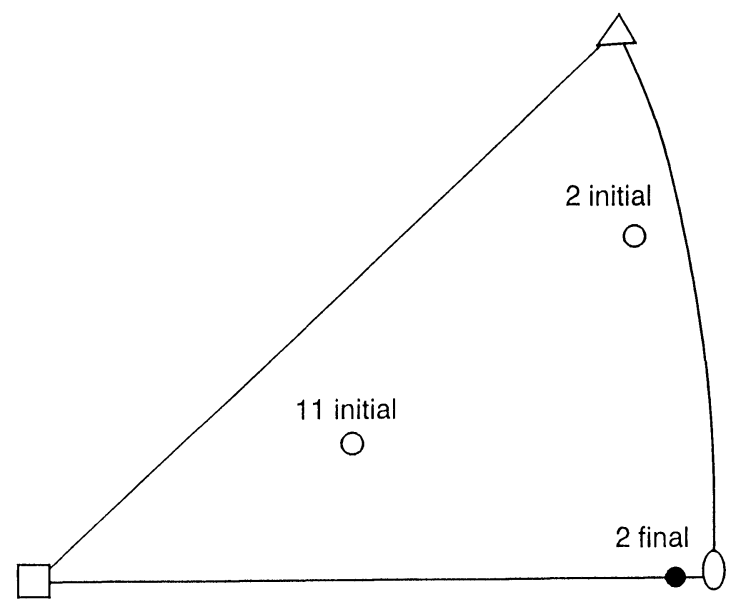

Figure 2 The orientation of compression axes of the crystals 2 and 11 whose behaviour are discussed in this paper. 

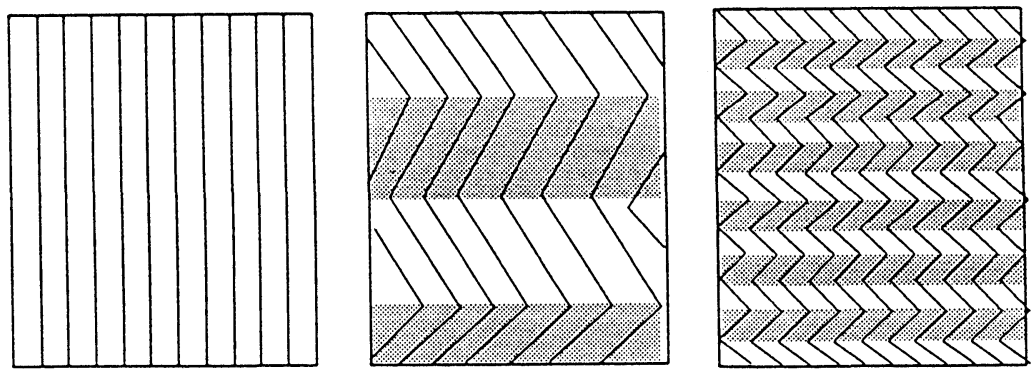

Figure 3 Schematic diagram of the formation of deformation bands.

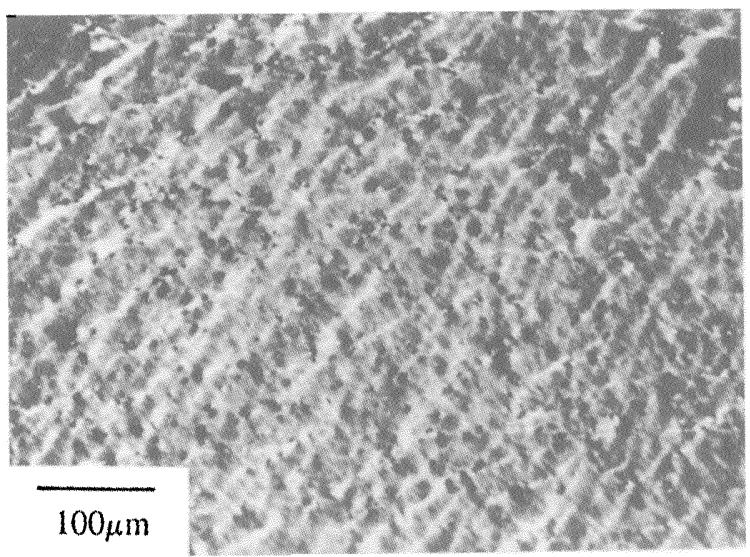

(a)

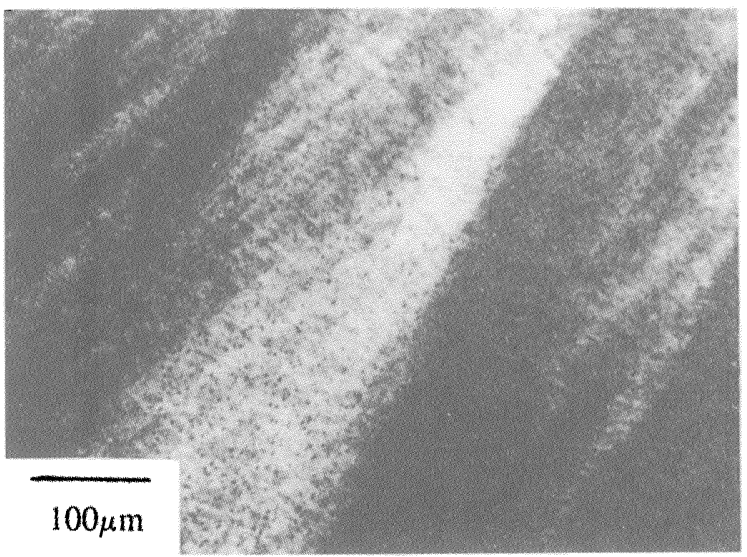

(b)

Figure 4 Optical micrographs showing deformation bands in crystal of orientation 11 deformed $50 \%$. a) Crystal SCL11 ( $5 \mu \mathrm{m}$ particles) b) Crystal SCS11 (0.7 $\mu \mathrm{m}$ particles). 
ments in a crystal of the same orientation, but containing large particles (SCL2). The finer scale of the bands is confirmed, and the misorientations are seen to be smaller $\left(\sim 15^{\circ}\right)$, Figure $6 \mathrm{~b}$.

The absolute orientations of the deformation bands in crystal SCS2 is presented in the 111 pole figures obtained by EBSP shown in Figure 7. In these figures, the

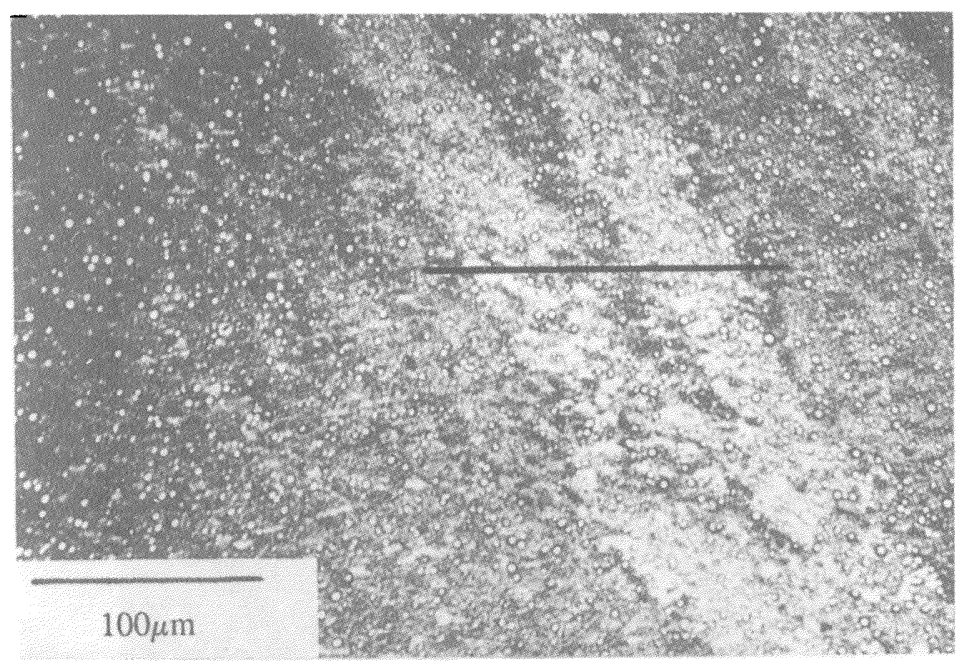

(a)

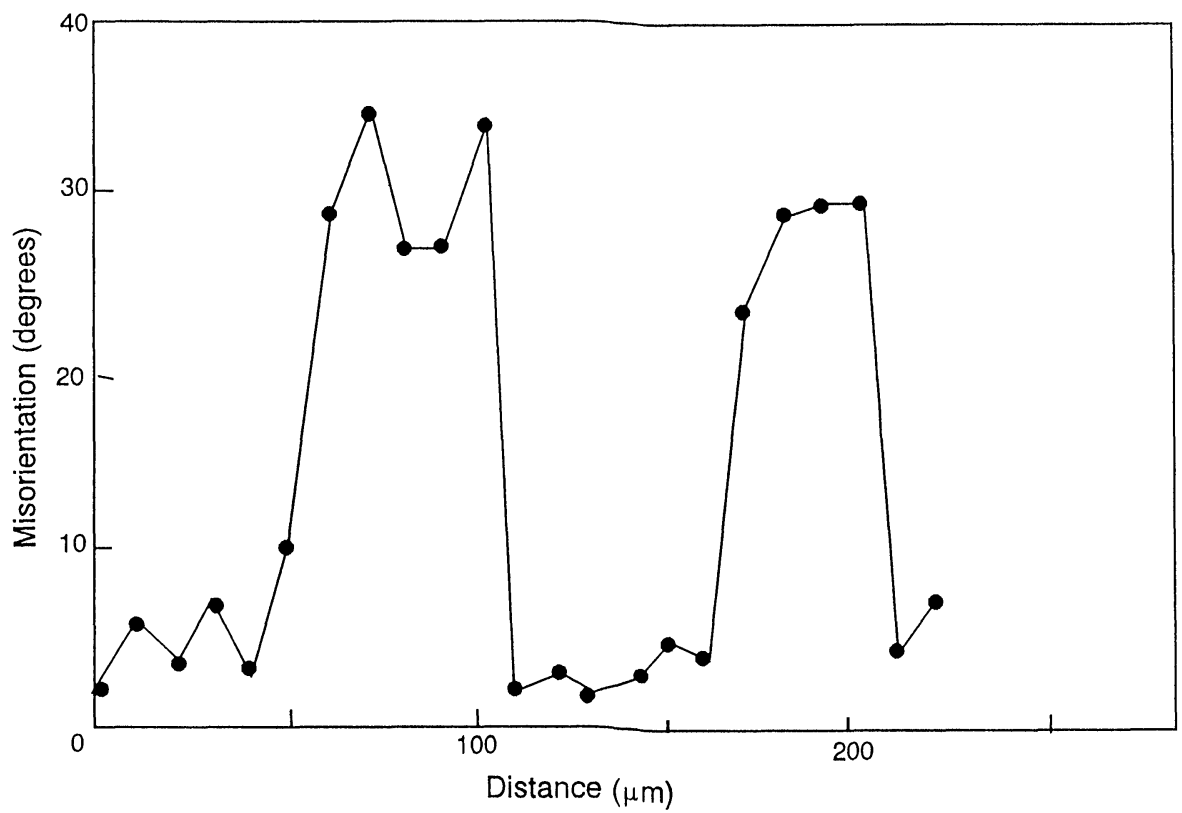

(b)

Figure 5 Deformation bands in crystal SCS2 $(0.7 \mu \mathrm{m}$ particles $)$ deformed $50 \%$. a) Scanning electron micrograph. b) Misorientations (relative to a fixed reference orientation) determined by EBSP along the line in a. 


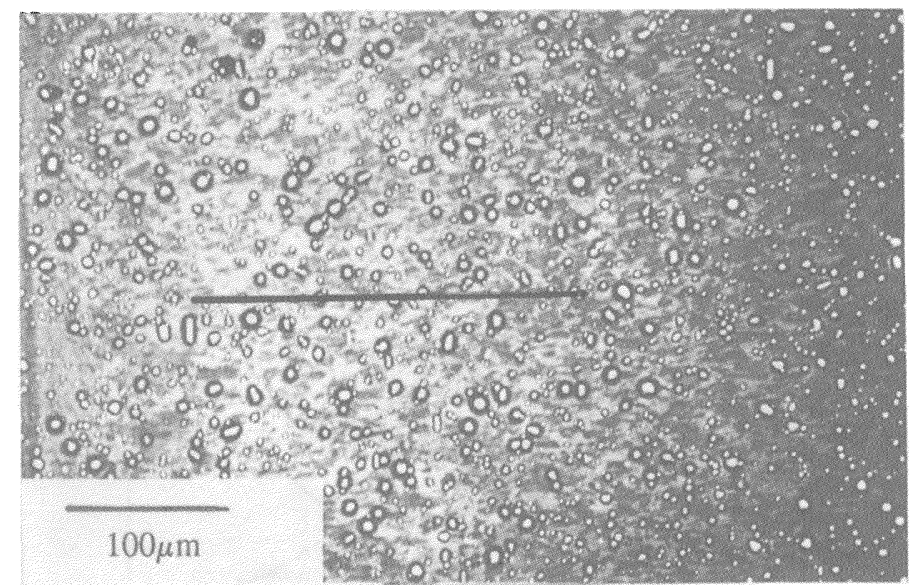

(a)

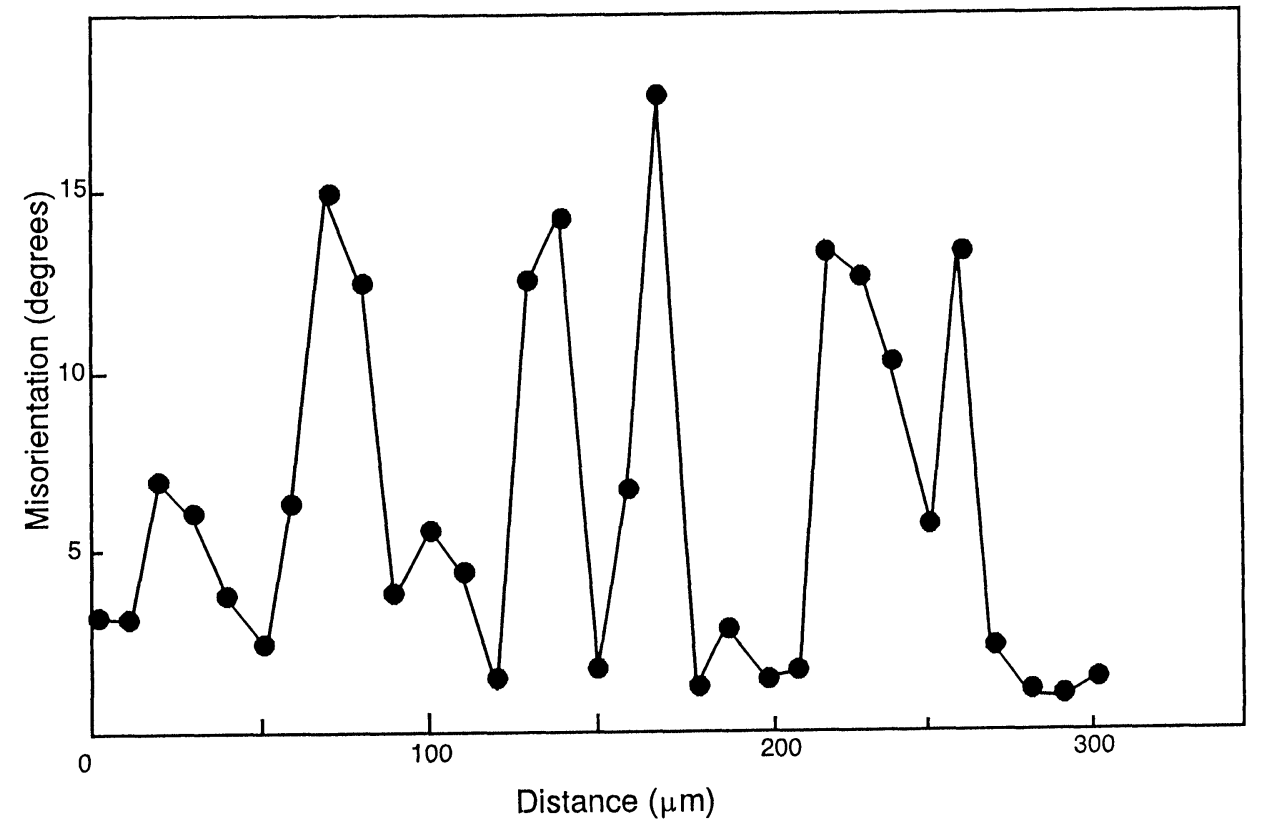

(b)

Figure 6 Fine deformation bands in crystal SCL2 (5 $\mu \mathrm{m}$ particles) deformed $50 \%$. a) Scanning electron micrograph. b) Misorientations determined by EBSP along the line in a.

centre of the pole figure corresponds to the compression axis. Figure 7a shows the total spread of orientations found across the sample. Figures $7 b$, c, and $d$ show how these orientations divide into three fairly sharp orientations, corresponding to the well characterised deformation bands in this material. The equivalent results for crystal SCL2 are shown in Figure 8. Compared to Figure 7a, the spread of orientations is somewhat smaller. However, the orientations of this figure could not be separated into clearly defined orientation components. 


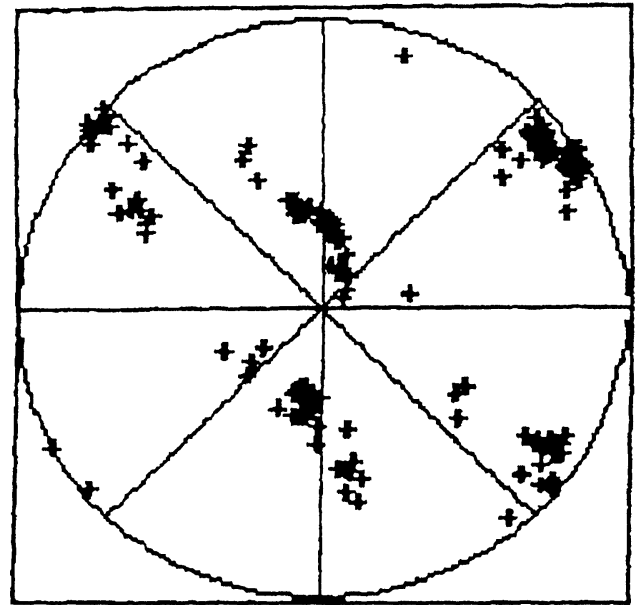

(a)

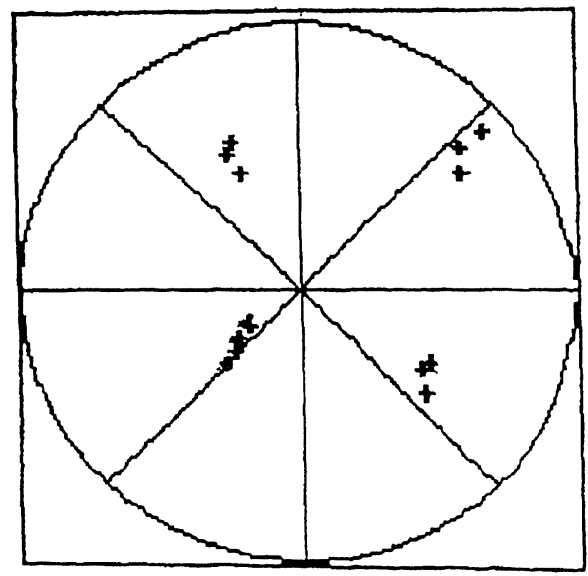

(c)

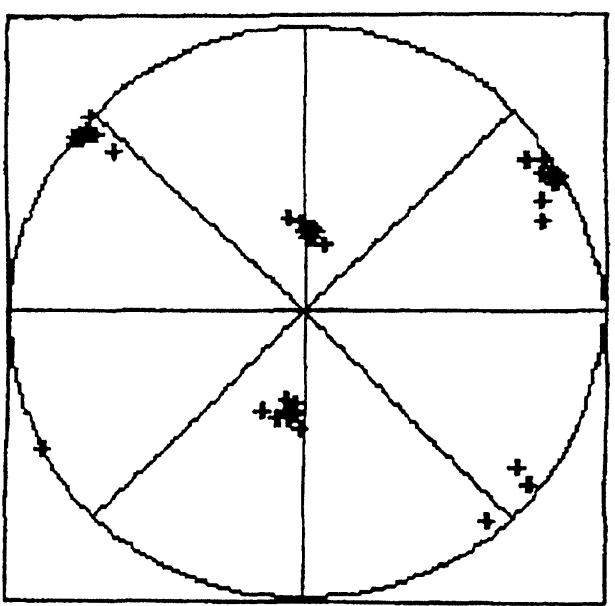

(b)

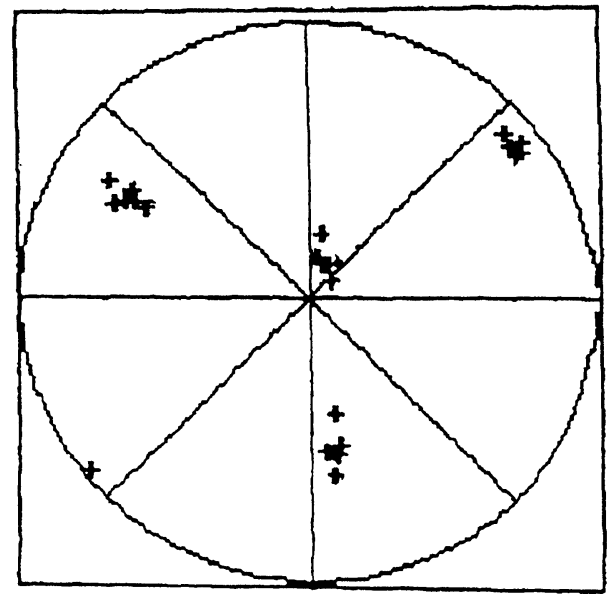

(d)

Figure 7 Orientations within the deformation bands in crystal SCS2. Data are presented as 111 pole figures with the compression axis in the centre. a) Data from a large area of the crystal. b) to d) The data are seen to divide into well defined orientation groups.

A few isolated large particles $(d \sim 20 \mu \mathrm{m})$ were found in the crystal containing the small particles, and as shown in Figure 9, there is a clear association between the deformation bands and the large particles. The results presented here suggest that the large particles act as nucleation sites for the deformation bands. Whether this is due to the dislocation activity in the region of the particles during plastic relaxation (Humphreys and Kalu, 1990) or whether it is due to the large particles affecting the slip distribution in the matrix is not yet known. 


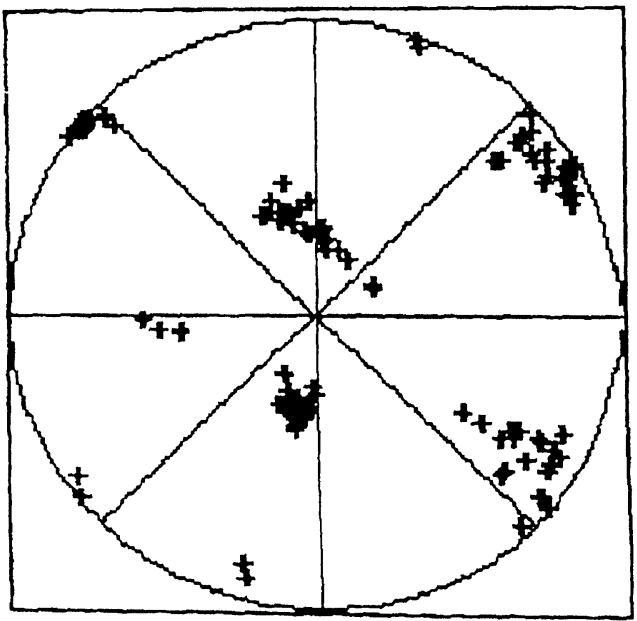

Figure 8 Orientations from crystal SCL2 presented as a 111 pole figure.

\section{RECRYSTALLIZATION}

\section{Polycrystals-Cold Rolled and Annealed}

Microstructures. The microstructures developed in polycrystals which were annealed after cold rolling $85 \%$ are shown in Figures 10 and 11. Figure 10a shows the initial stages of recrystallization in an alloy containing large $(6 \mu \mathrm{m})$ particles (PCL). Particle stimulated recrystallization is seen to occur, and the fully recrystallized alloy (Figure 10b) has a structure of small equiaxed grains. Figure 11 shows the recrystallized microstructure of a polycrystal containing smaller $(1.5 \mu \mathrm{m})$ particles (PCS), which is almost fully recrystallized. In this material, particle stimulated nucleation occurs only at particles in the prior grain boundaries, where the particles are slightly larger, and in these areas a structure

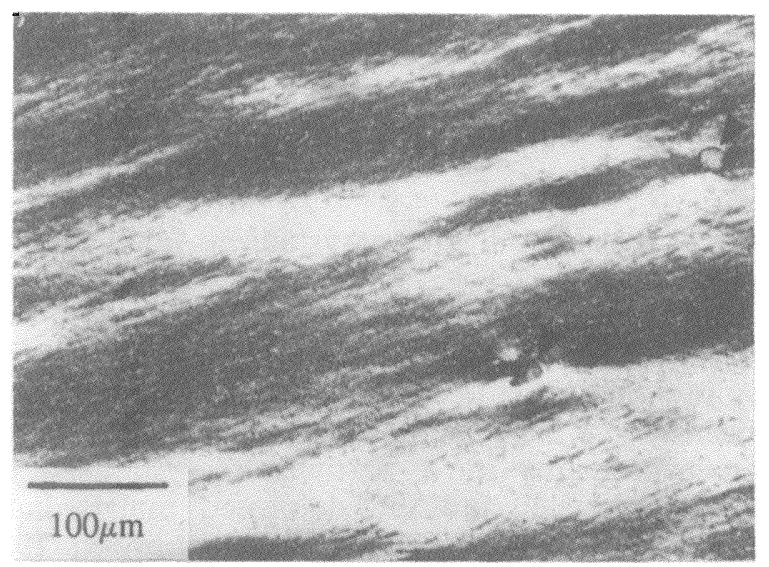

Figure 9 Optical micrograph of deformation bands in crystal SCS2. Association of large $(20 \mu \mathrm{m})$ particles and deformation bands is arrowed. 


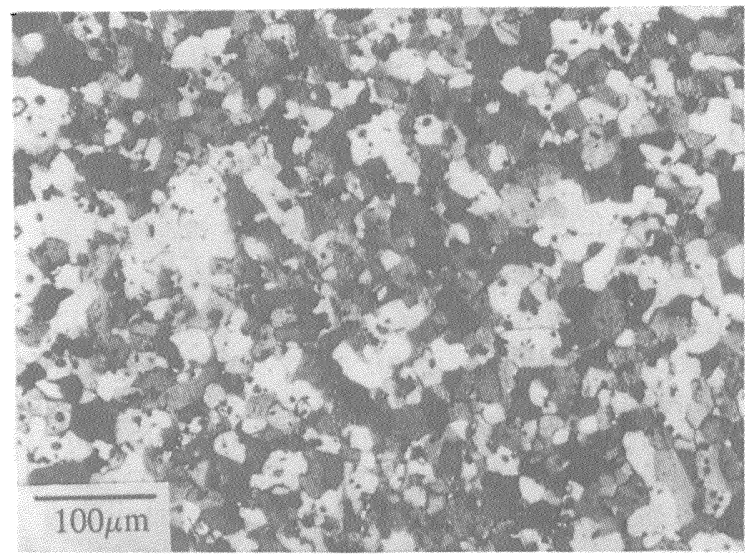

(a)

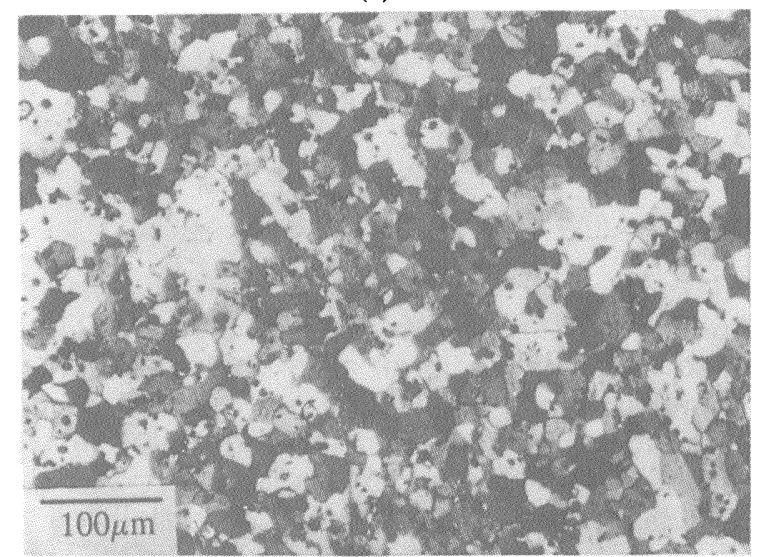

(b)

Figure 10 Recrystallization in deformed polycrystal PCL. a) Particle stimulated nucleation during the early stages of recrystallization. b) Fully recrystallized specimen.

of small equiaxed grains is found (line A-B). In the rest of the material, the grains are larger and often elongated.

Textures. The 111 pole figure from the recrystallized alloy PCL, in which PSN occurred is shown in Figure 1c. The texture is best described as a weakened rolling texture in which the peaks have fallen from $4.8 \times$ random to $3.2 \times$ random.

It has been observed by several workers that the texture in a rolled polycrystalline alloy in which PSN occurs is not random, but is a spread of the rolling texture (Jensen et al., 1985 Humphreys and Jensen, 1986, Jensen et al., 1987). However, measurements in a partially recrystallized sample of the relationship between the nuclei and the deformed matrix indicate that the misorientations are generally large (Chan and Humphreys, 1984). Figure 12 confirms this, and shows that the misorientations generally lie between 10 and $50^{\circ}$. The question then arises as to why with such large misorientations between 


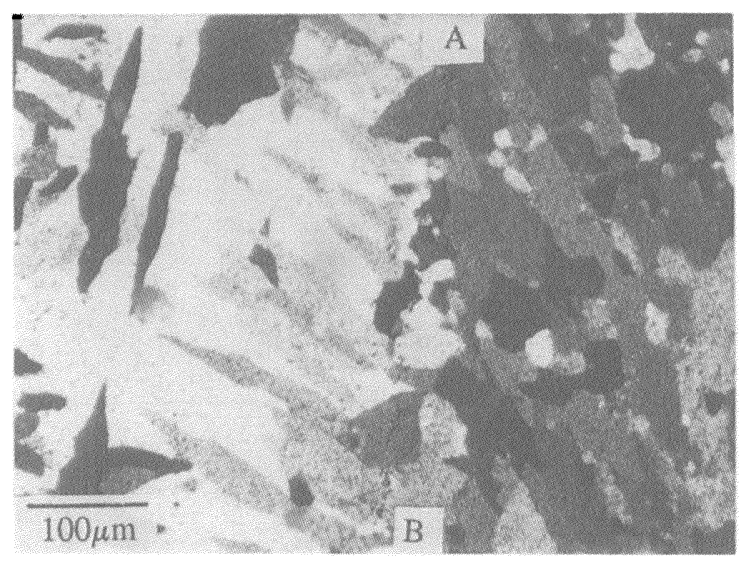

Figure 11 Recrystallized specimen PCS showing smaller grain formed at the prior grain boundary A-B.

deformed and recystallizing material we obtain such a relatively sharp recrystallization texture. It has been suggested (e.g. Jensen et al., 1986) that competing recrystallization mechanisms other than PSN may be responsible for the large retained component of the rolling texture.

In order to test this hypothesis, a simple computer model of PSN textures has been developed. "Grains" of the orientation components which comprise the deformation texture of aluminium were generated in a computer, and the spread around these orientations was chosen to be such as to produce a 111 pole figure of similar strength to the experimental one of Figure 1a. Individual orientations in

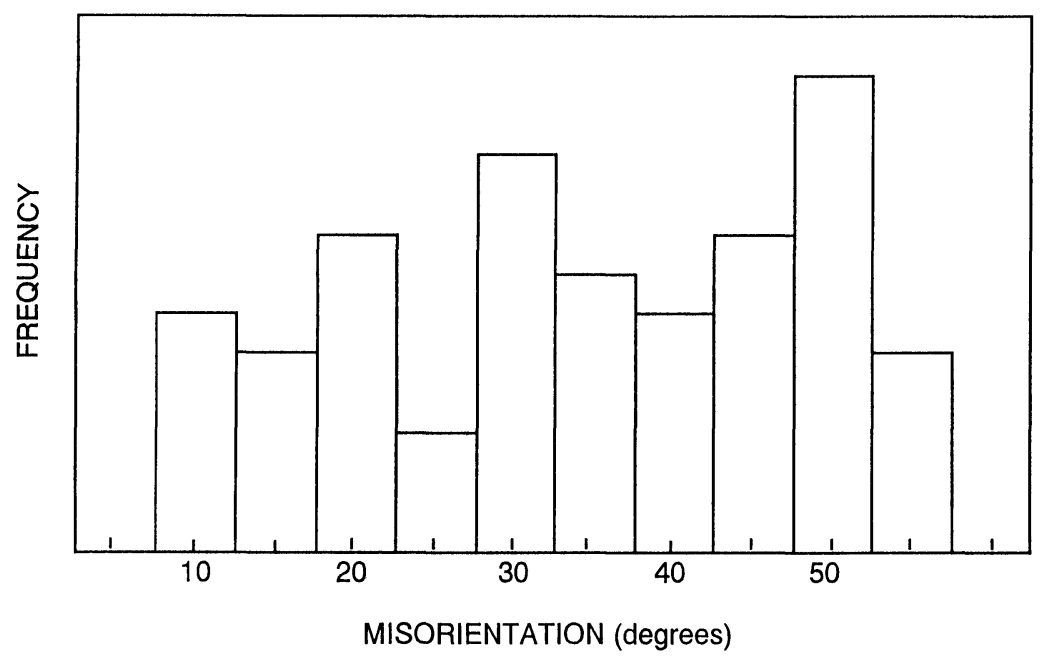

Figure 12 The misorientations between PSN nuclei and adjacent matrix determined by EBSP. 


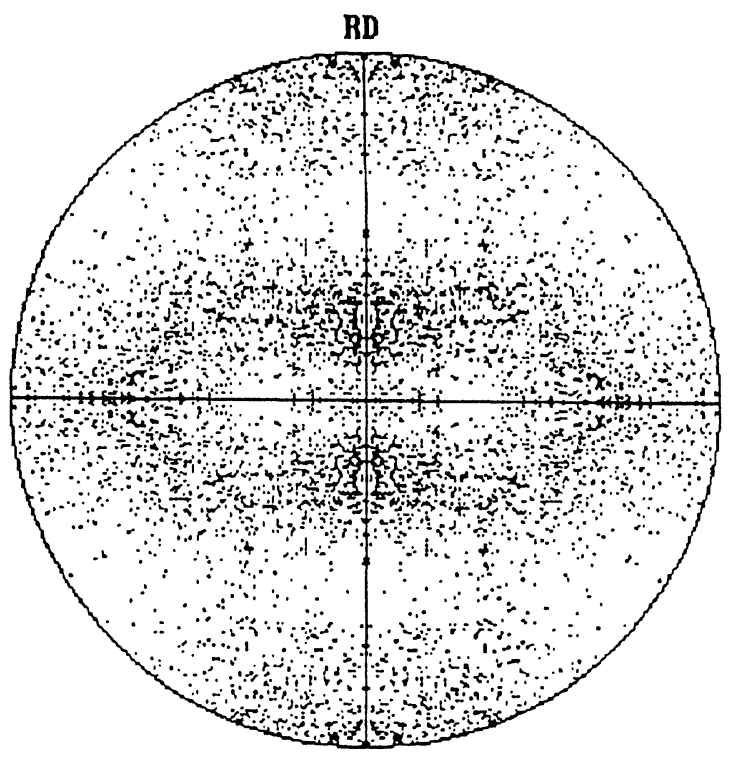

(a)

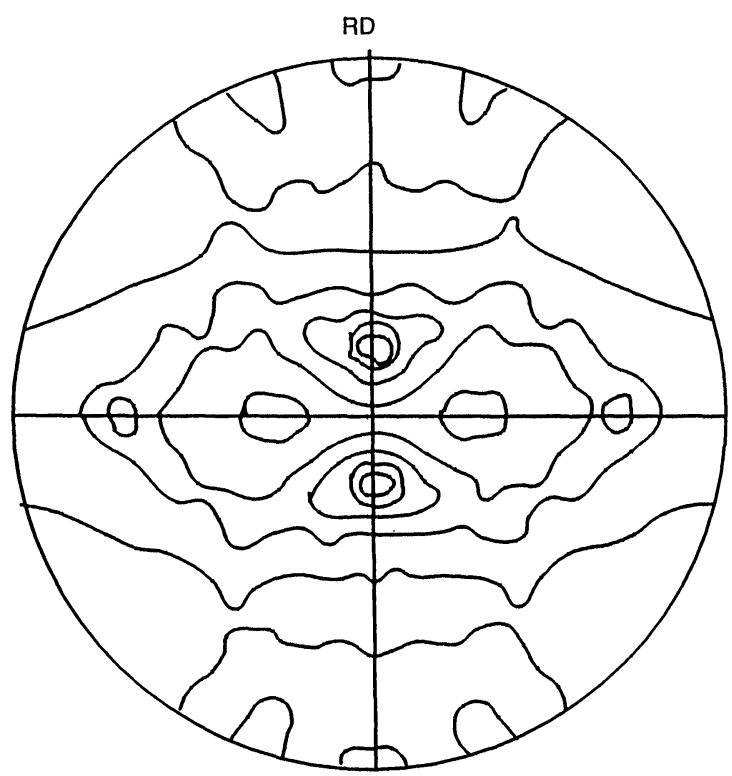

(b)

Figure 13 Computer model of the deformation and PSN textures. a) 111 pole figure of the individual orientations in the deformed specimen. b) Contour plot of a) at intervals of $1 \times$ random. c) "PSN" texture showing some retained rolling components. 


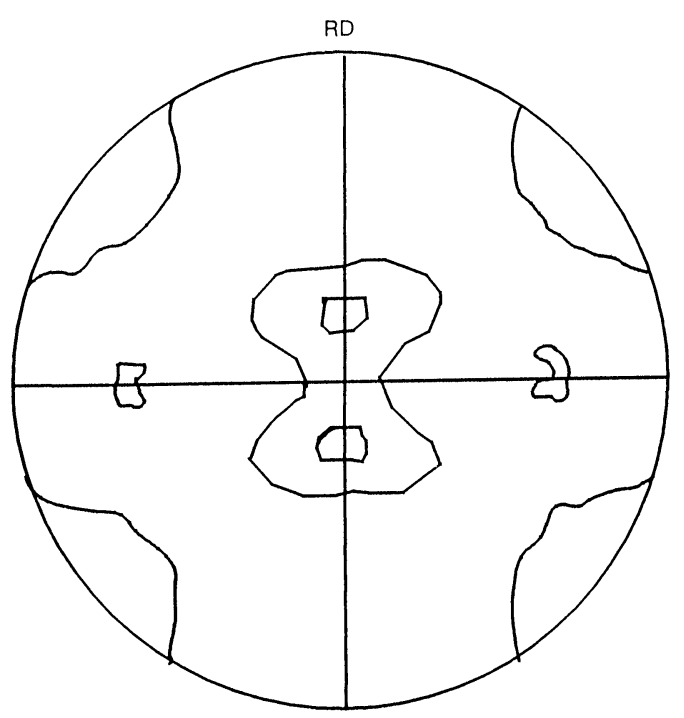

(c)

Figure 13 Computer model of the deformation and PSN textures. a) 111 pole figure of the individual orientations in the deformed specimen. b) Contour plot of a) at intervals of $1 \times$ random. c) "PSN" texture showing some retained rolling components.

such a model are shown in Figure 13a, and a contour plot for a 10,000 grains is shown in Figure 13b.

To represent the grains nucleated by PSN, each of the grains in the deformed structure was allowed to nucleate 100 grains whose orientations deviated (randomly) by between $5^{\circ}$ and $45^{\circ}$ from the parent grain. Rotations about random axes or about specific axes such as $\langle 111\rangle$ were investigated, although no marked effect of rotation axis was noted. The resultant polefigure is shown in Figure 13c. It is noticeable that there is still a considerable component of the rolling texture and that the drop in peak intensity from $5.4 \times$ random to $3.1 \times$ random is comparable with that found experimentally. This model suggests that the observed weak rolling textures found in materials which have recrystallized by PSN are explainable simply in terms of the spread of orientations generated by PSN and do not necessarily imply the additional operation of any other recrystallization mechanisms.

\section{Single Crystals-Cold Rolled and Annealed}

In a heavily rolled polycrystal, the rotations at the particles, which are a direct consequence of the operative slip systems (Humphreys and Kalu, 1990), are complex, and there are many possible nucleus orientations available. In contrast, in a single crystal deformed in uniaxial compression, the number of slip systems operating will be limited, and therefore the spread of orientations in the deformation zones and hence the number of nuclei orientations will be small.

A crystal containing $6 \mu \mathrm{m}$ particles (SCL2), deformed $50 \%$ in compression was annealed so as to partly recrystallize. Metallography confirmed that particle 


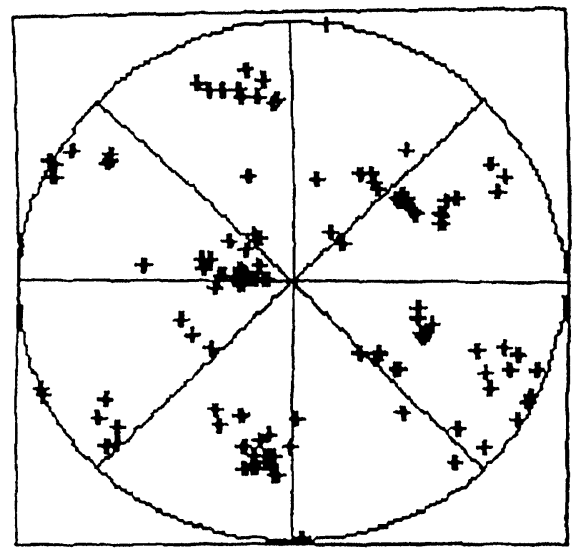

(a)

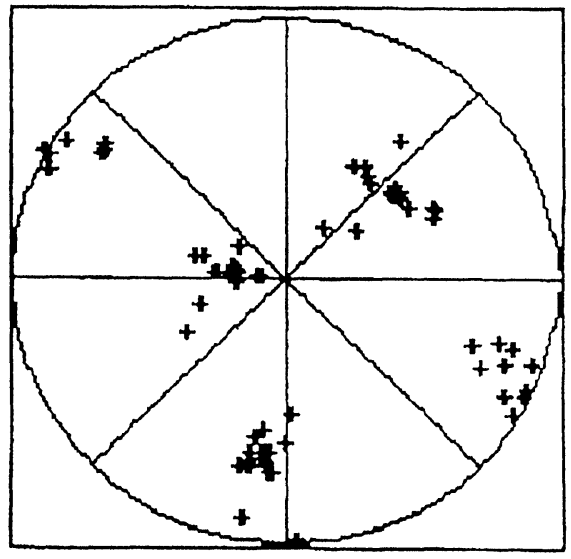

(b)

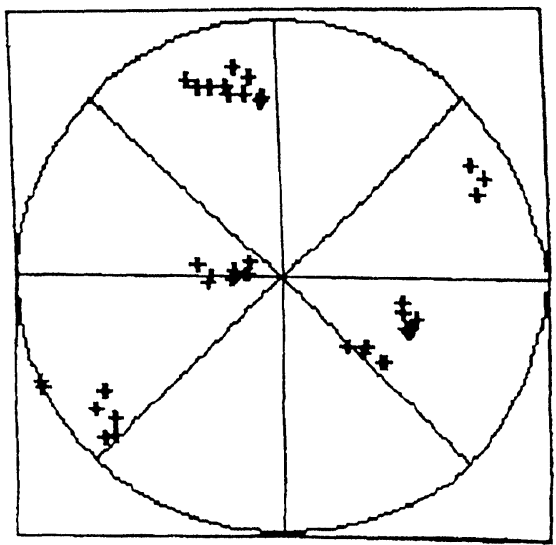

(c)

Figure 14 The 111 poles of PSN grains in single crystal SCL2 deformed $50 \%$ and annealed. a) All nuclei b) and c) show two prominent components of the orientation.

stimulated nucleation occurred. The orientations of the small recrystallized grains, were measured by EBSP and are shown in Figure 14a. The majority of these orientations fall into two well defined orientation groups as shown in Figures $14 \mathrm{~b}$ and $14 \mathrm{c}$. The mean orientation of each of these groups and the relationship with the deformed matrix is shown in Figure 15a and $b$, and the axes of rotation between the matrix and the recrystallized grains is shown in Figure 16.

The orientation of a grain originating by particle-stimulated nucleation is expected to be an orientation present in the deformation zone adjacent to the particle. It has been shown that in particle-containing single crystals, for the operation of a single slip system $(h k l)[u v w]$, the orientations within the deformation zone are related to the matrix by a rotation about the axis $h k l^{\wedge} u v w$ (Humphreys 1979). Recent work on deformed particle-containing polycrystals is consistent with this model (Humphreys and Kalu 1990). 


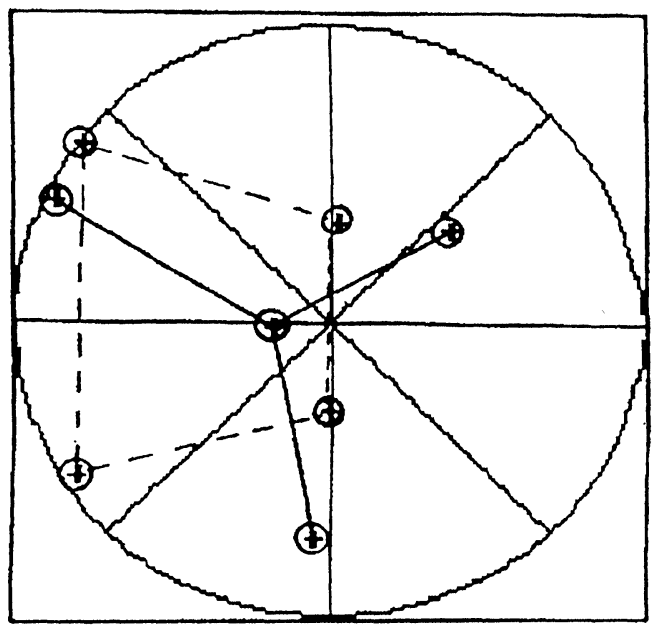

(a)

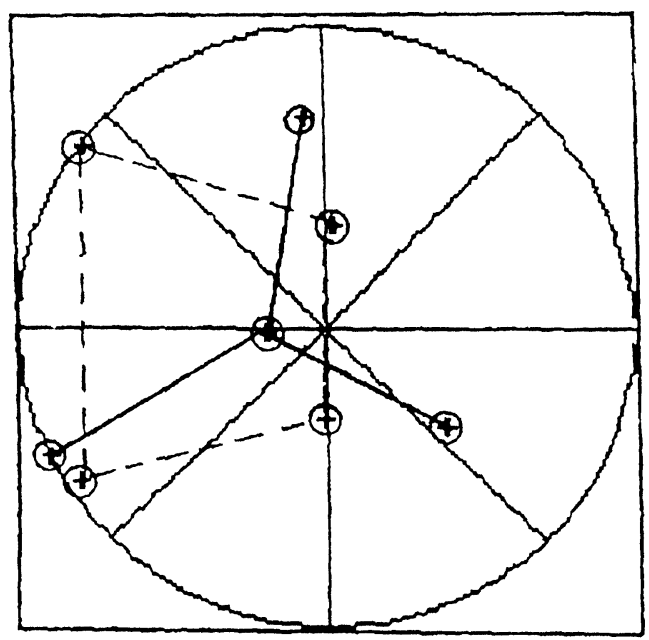

(b)

Figure 15 The orientations, represented by 111 poles, of the two recrystallization texture components measured in Figure 14, compared to the orientation of the matrix, whose orientation is shown dotted.

For crystal 2, whose initial and final orientations are shown in Figure 2, the predicted slip systems, shear strains and rotation axis are shown in Table 2 . The predicted rotation axes are compared in Figure 16 with those found experimentally. The agreement between the theory and experiment is good, suggesting that the model is valid for single crystals deforming on two slip systems. We detected no cases of nucleation of more than one grain at any particle, and assume that the

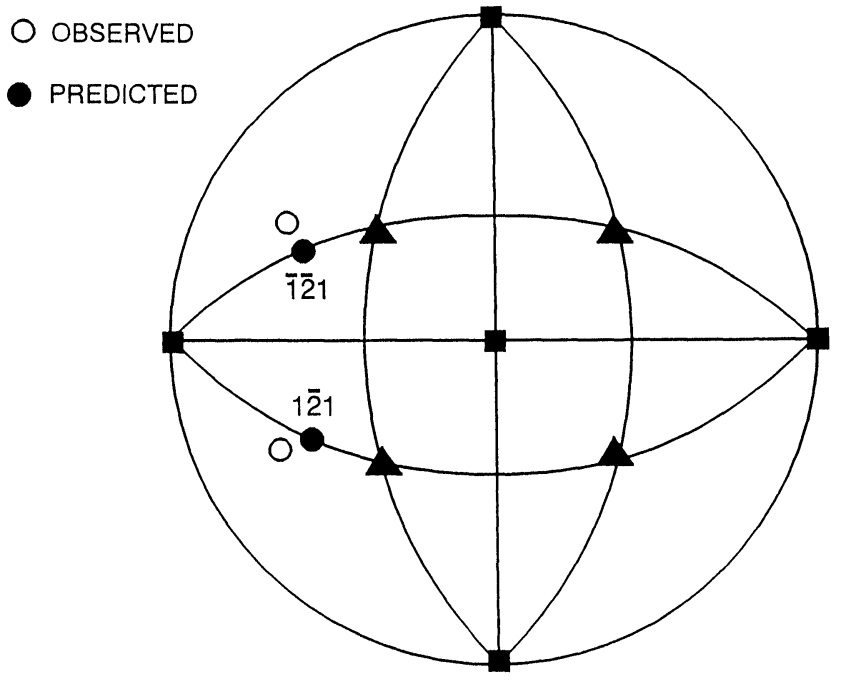

Figure 16 Comparison of the measured rotation axes with those predicted. 
Table 2 The deformation of crystal of orientation 2

\begin{tabular}{llll}
\hline & Slip system & Rotation axis & Shear strain \\
\hline Primary & $(111)[\overline{1} 01]$ & {$[1 \overline{2} 1]$} & 0.85 \\
Secondary & $(\overline{1} 11)[101]$ & {$[\overline{1} 1]$} & 0.49 \\
\hline
\end{tabular}

favouring of one orientation of nucleus over the other must be due to local fluctuations in slip activity.

The results discussed above clearly show how a strong recrystallization texture can be produced by PSN if the deformation is such as to restrict the number of deformation zones and hence possible nucleus orientations. In the case of the heavily deformed polycrystals discussed earlier, then the weakened deformation texture is explained by the greater choice of nucleus orientations.

\section{CONCLUSIONS}

(1) Particles influence the scale of deformation bands in Al-Si. Large particles nucleate the bands and hence give rise to fine scale bands, whereas small $(<1 \mu \mathrm{m})$ particles have little effect on deformation band formation.

(2) The recrystallization texture of a cold rolled polycrystal containing large $(6 \mu \mathrm{m})$ particles is a weakened rolling texture. It is shown that this is consistent with particle stimulated nucleation with orientations rotated by up to around $45^{\circ}$ from the matrix.

(3) The recrystallization texture of a single crystal deformed on two slip systems is sharp and is accounted for by nucleation from a restricted number of deformation zones. The rotation axes are consistent with a model for the plasticity of two-phase alloys.

\section{References}

Becker, R., Butler, J. F., and Lalli, L. (1991). Met. Trans. 22A, 45.

Chan, H. M. and Humphreys, F. J. (1984). Metal Science 18, 525.

Dingley, D. J. (1984). Scanning Electron Microscopy, 11, 569.

Dillamore, I. L. and Katoh, H. (1974). Metal Sci. 8, 73.

Humphreys, F. J. (1977). Acta Met. 25, 1323.

Humphreys, F. J. (1979). Acta Met. 27, 1801.

Humphreys, F. J. and Jensen, D. J. (1986). Proc. 7th Int. Riso. Symp. Riso, Denmark. Ed. Hansen. 93.

Humphreys, F. J. and Kalu, P. N. (1990). Acta Met. 38, 917.

Jensen, D. J., Hansen, N. and Humphreys, F. J. (1985). Acta Met. 33, 2155.

Jensen, D. J., Hansen, N. and Humphreys, F. J. (1987). Proc. 8th Int. Conf. on Textures. Santa Fe, USA. Ed. Kallend \& Gottstein. TMS. 431. 